\title{
Bronquiolitis en una Zona de Salud urbana: Factores demográficos y medioambientales
}

\author{
Candelaria Ayuso Rayaa , Ana Castillo Serranob, \\ Francisco Escobar Rabadán ${ }^{c}$, Josefa Plaza Almeida ${ }^{d}$
}

${ }^{a}$ Residente de Medicina Familiar y Comunitaria. Unidad Docente de Albacete.

${ }^{\mathrm{b}}$ Residente de Pediatría. Hospital General Universitario de Albacete.

'Médico de Familia. Centro de Salud Universitario Zona IV de Albacete. Profesor Asociado de la Facultad de Medicina de Albacete.

dPediatra. Centro de Salud Zona VIII de Albacete.

Correspondencia: Candelaria Ayuso Raya, Centro de Salud Zona IV, c/ Seminario $n^{\circ} 4$, 02006 Albacete, España. Telf.: 967510094, correo electrónico:

candeayuso@hotmail.com.

Recibido el 23 de marzo de 2010.

Aceptado para su publicación el 26 de mayo 2010.

\section{RESUMEN}

Objetivo. Determinar qué factores externos condicionan el desarrollo de bronquiolitis y son modificables en la práctica clínica diaria.

Diseño del estudio. Estudio caso-control.

Emplazamiento. Atención Primaria.

Participantes. 34 lactantes (1-24 meses) diagnosticados de bronquiolitis entre octubre 2008 a abril 2009. Grupo control de 32 lactantes que no habían padecido bronquiolitis.

Resultados. El 50\% de los casos eran menores de 7 meses, con un leve predominio masculino (19). En el análisis bivariante no se encontraron diferencias significativas entre casos y controles para las variables: edad gestacional, peso al nacimiento, edad materna, asistencia a guardería y tabaquismo en domicilio. Existía una patología de base en el $47 \%$ de los casos frente al $13 \%$ de los controles $(p=0,002)$. El $45 \%$ de los casos no tomaba lactancia materna, frente a un $15,6 \%$ de los controles $(p=0,045)$. Por medio de un análisis de regresión logística se confirmó la independencia de estas variables, con valores de OR: 0,25 (IC 95\%: 0,07-0,94), 4,53 (IC 95\%: 1,12-18,32), 11,1(IC 95\%: 1,22-100,52) respectivamente para lactancia, enfermedades de base e ingresos previos.

Conclusiones. La lactancia materna es un claro factor protector, siendo su promoción una de la actividades más eficaces para evitar la bronquiolitis. La presencia de patología de base u hospitalización previa predispone al desarrollo de bronquiolitis y es, por tanto, fundamental el seguimiento estrecho de estos lactantes de riesgo en época de epidemia. Palabras clave. Bronquiolitis, Factores de Riesgo, Lactancia Materna.

\section{ABSTRACT}

Bronchiolitis in urban health areas: demographic and environmental

Objective. To determine what factors effect the development of bronchiolitis and can be modified in daily clinical practice.

Study design. Case-control study.

Setting. Primary care

Participants. 34 infants (1-24 months) diagnosed with bronchiolitis between October 2008 and April 2009. A control group of 32 infants who did not have bronchiolitis.

Results. Fifty percent of infants were less than 7 months old and predominantly male (19). The bivariate analysis did not reveal any significant differences between the patients and controls as regards: gestational age, birth weight, maternal age, attending day-care centres, smoking in the home. There was underlying disease in $47 \%$ of patients compared to $13 \%$ of controls $(p=0.002)$. Forty five percent of the patients were not lactating compared to $15.6 \%$ of controls $(p=0.045)$. The independence of these variables was confirmed by logistic regression analysis with OR values: 0.25 (95\% Cl: 0.07-0.94), 4.53 (95\% Cl: $1.12-$ $18.32), 11.1$ (95\% Cl: $1.22-100.52)$ for lactating, underlying disease and previous hospital stays, respectively.

Conclusions. Breast-feeding is clearly a protective factor. Encouraging breast-feeding is one of the most effective actions in preventing bronchiolitis. The presence of an underlying disease or previous hospital stays predisposes infants to developing bronchiolitis and it is therefore essential to closely monitor infants at risk in the epidemic periods.

Key words. Bronchiolitis, Risk Factors; Breast Feeding.

Una versión preliminar de este trabajo fue presentada como comunicación en póster en el XIX Congreso Español de Pediatría Social: "Infancia y Diversidad: presente y futuro", celebrado en Sevilla los días 15, 16 y 17 de Octubre de 2009. 


\section{INTRODUCCIÓN}

La bronquiolitis es una infección aguda del tracto respiratorio que provoca el primer episodio de broncoespasmo en un lactante. Fue descrita como entidad clínica aislada originariamente en 1941 tras una epidemia de gripe en el Reino Unido'. Esta enfermedad tiene una repercusión importante en nuestro sistema sanitario y constituye una causa muy frecuente de consulta en Pediatría durante los meses de noviembre a febrero.

La etiología más frecuente de la bronquiolitis es el virus respiratorio sincitial (VRS), causante del 20$40 \%$ de los casos. En época epidémica hasta el $60 \%$ en los hospitalizados están relacionados con él. En el hemisferio norte es más frecuente entre noviembre-abril, con un pico estacional en enerofebrero ${ }^{2}$. Hay muchos otros virus que pueden causar bronquiolitis, algunos como el Metapneumovirus (hMPV) en coinfección con el VRS parece relacionarse con una mayor gravedad de la enfermedad. Otros por orden de importancia son: Parainfluenza, Adenovirus, Influenza, Rinovirus, Coronavirus, Metapneumovirus Humano y Bocavirus Humano. El Mycoplasma en niños mayores y la Chlamydia pueden dar cuadros similares ${ }^{2,3}$.

EI VRS es la primera causa de infección respiratoria aguda grave en forma de bronquiolitis o neumonía, y además es la principal causa de hospitalización por infección del tracto respiratorio inferior en menores de 2 años en los países desarrollados. La morbilidad y la mortalidad relacionadas con la bronquiolitis y el VRS son mayores en prematuros, en las displasias broncopulmonares (DBP), en las cardiopatías congénitas hemodinámicamente significativas y en algunas inmunodeficiencias. EI VRS es relativamente inestable en condiciones ambientales, se inactiva por detergentes y por diversos desinfectantes y puede transmitirse por fomites y por vía respiratoria. Cada año alrededor del 10\% de los lactantes tienen bronquiolitis. Se produce un pico entre los 2 y los 6 meses de edad.

Además de los datos anteriores, la gran repercusión de esta enfermedad se debe a que entre el $50-70 \%$ de los niños con bronquiolitis tendrán episodios de sibilancias recurrentes en los meses e incluso en años posteriores ${ }^{2}$. En España la tasa de hospitalización por VRS es de 35-37/1000 en lactantes de menos de 6 meses y de 25/1000 en menores de 12 meses $^{4}$.

Se han descrito otros criterios clínicos asociados a la enfermedad y definidos como: "sujetos menores de 2 años con aparición rápida de rinofaringitis (2448 horas) y asociación de alguno de los siguientes síntomas: disnea espiratoria con polipnea, tiraje subcostal, intercostal o supraesternal, distensión torácica (clínica o radiológica), dificultad respiratoria y/o sibilancias y/o crepitantes de predominio espiratorio y primer episodio coincidiendo con el periodo epidémico del VRS".

Los factores de riesgo asociados a una morbimortalidad más alta en un $30 \%$ de los casos se corresponderían con una edad inferior a 3 meses (especialmente menores de 6 semanas), lactantes con enfermedad pulmonar crónica tales como la Fibrosis Quística, la displasia bronco-pulmonar, la malformación pulmonar, las cardiopatías congénitas hemodinámicamente significativas, inmunodeficiencias, enfermedades neuromusculares o metabólicas, la prematuridad con un peso al nacimiento inferior a 2500 gramos y la desnutrición ${ }^{5}$.

A pesar de su elevada prevalencia, no supone un grave riesgo para los lactantes sanos, que suelen mejorar de forma espontánea o con mínimas medidas de soporte. Los casos de infección grave que pueden requerir cuidados intensivos están casi siempre relacionados con uno o varios factores predisponentes, tales como la lactancia artificial, la asistencia a guardería, el hacinamiento y el tabaquismo familiar $5,6,7$.

No conocemos la existencia de estudios epidemiológicos previos, en relación con este tema, realizados en nuestro medio. Dado el interés creciente que suscita esta patología debido a su elevada prevalencia, constituyendo uno de los principales motivos de consulta tanto en el ámbito ambulatorio como en el hospitalario, y ante la ausencia de una estrategia terapéutica clara, pretendemos determinar qué factores externos condicionan su desarrollo y son modificables en nuestra práctica clínica diaria.

\section{MATERIAL Y MÉTODO}

El estudio fue realizado en la consulta de Pediatría del Centro de Salud Zona I de Albacete. Se trata de un estudio retrospectivo de casos y controles, en el que se incluyeron 34 lactantes que fueron diagnosticados de bronquiolitis entre octubre de 2008 y abril de 2009 y un grupo control de 32 lactantes que no habían sufrido esta patología, con edades comprendidas entre 0-24 meses. Se excluyeron del grupo de control aquellos pacientes con patología respiratoria similar al proceso infeccioso a estudio.

Para el diagnóstico se emplearon criterios clínicos, aplicándose la puntuación clínica de Wood-Downess modificada por Ferres ${ }^{2}$, y puntualmente se solicitó radiografía de tórax. Para conocer el grado de exposición al tabaco y otros posibles factores de riesgo asociados, se recogió la información de los padres mediante una entrevista clínica, realizada por la Pediatra, en la que se incluían la edad del niño, edad de la madre, la edad gestacional, el peso del recién nacido, la edad al diagnóstico, la asistencia a guardería, lactancia materna (LM), artificial o mixta, el motivo del ingreso, la existencia de hábito 
tabáquico materno y paterno en domicilio, las enfermedades previas, los ingresos hospitalarios previos al diagnóstico, la existencia de ingresos previos por bronquiolitis, el tiempo de hospitalización, la gravedad del cuadro, el tratamiento recibido, la identificación o no del VRS como agente causal, si se realizó la vacunación frente a VRS, la fecha del diagnóstico y duración del cuadro.

La medida de asociación utilizada fue la odds ratio (OR), calculando el intervalo de confianza (IC) del $95 \%$. Se realizó un análisis bivariante, por medio del test de chi cuadrado, para el contraste de variables cualitativas y la prueba $t$ de Student para el contraste de variables continuas, recurriendo a test no paramétricos si éstas no presentaban distribución normal. El análisis estadístico se completó con una regresión logística, siguiendo una estrategia hacia atrás partiendo del modelo máximo, extrayendo aquellas variables independientes cuyo coeficiente de regresión tenía una $p<0,05$. Se empleó el programa SPSS 12.0.

\section{RESULTADOS}

El $50 \%$ de los pacientes diagnosticados de bronquiolitis tenía una edad igual o inferior a 7 meses, con leve predominio masculino (55,9\%), como podemos ver en la tabla 1. Respecto al tratamiento, la opción más empleada fue salbutamol en cámara espaciadora hasta en dos tercios de los casos. El controvertido uso de corticoides orales en el proceso agudo estaba registrado en 7 casos (tabla 2).

La edad gestacional y el peso al nacimiento fueron menores en los casos (figura 1), aunque las diferencias no eran significativas. La edad materna, con un rango de 17 a 44 años, fue superponible en ambos grupos (tabla 1). Tampoco se encontraron diferencias significativas para las variables asistencia a guardería y tabaquismo en domicilio.

Como podemos observar en la figura 2 , el $45 \%$ de los casos no tomaba lactancia materna, frente a un $15,6 \%$ de los controles ( $p=0,045$; OR: 0,222 , IC 95\%: 0,069-0,719).

Al no ser detectado rutinariamente el antígeno VRS disponemos de datos insuficientes $(n=11)$ : 5 casos diagnosticados de bronquiolitis con VRS positivo versus 6 diagnosticados de bronquiolitis con VRS negativo, con sólo 1 caso de lactancia materna en cada grupo.

La patología de base, como cardiopatía congénita, patología respiratoria del recién nacido pretérmino (RNPT), reflujo gastro-esofágico (RGE) y fallo de medro, se hallaba presente en el $47 \%$ de los casos frente al $13 \%$ de los controles ( $p=0,002)$. Un 33\% presentaba un ingreso previo por motivos diferentes a la bronquiolitis.

\begin{tabular}{lccc}
\hline & Casos & Controles & Total \\
\hline Edad de la madre (años) & 31,5 & 33,0 & 32,0 \\
[mediana (RI)] & $(29,0-35,7)$ & $(31,0-34,7)$ & $(29,0-35,0)$ \\
Edad del niño (meses) & 8,5 & 8,0 & 7,5 \\
[mediana (RI)] & $(4,0-14,7)$ & $(2,2-18,0)$ & $(3,0-16,0)$ \\
Sexo & & & \\
$\quad$ Niña & 15 & 18 & 33 \\
Niño & 19 & 14 & 33 \\
\hline
\end{tabular}

Tabla 1. Distribución de frecuencias o medidas de tendencia central para las variables demográficas consideradas en el estudio, para las que no se encontraron diferencias significativas. RI: rango intercuartil.

\begin{tabular}{lr}
\hline Salbutamol en cámara & $68,1 \%$ \\
Adrenalina nebulizada y Propionato de Fluticasona & $4,5 \%$ \\
Salbutamol y Prednisolona esteaglato & $13,6 \%$ \\
Salbutamol y Propionato de Fluticasona & $4,5 \%$ \\
Salbutamol y corticoide oral & $4,5 \%$ \\
Suero salino fisiológico & $4,5 \%$ \\
\hline
\end{tabular}

Tabla 2. Porcentaje de pacientes con bronquiolitis que recibieron los principales tratamientos administrados en fase aguda y de mantenimiento. 

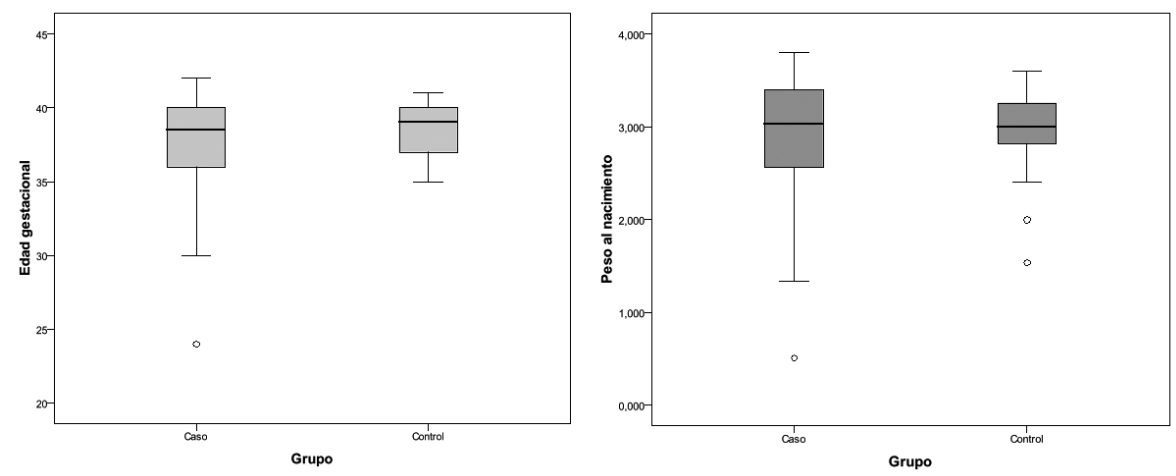

Figura 1. Distribución de los valores de semanas de gestación en que se produjo el nacimiento y el peso al nacer, para casos y controles.

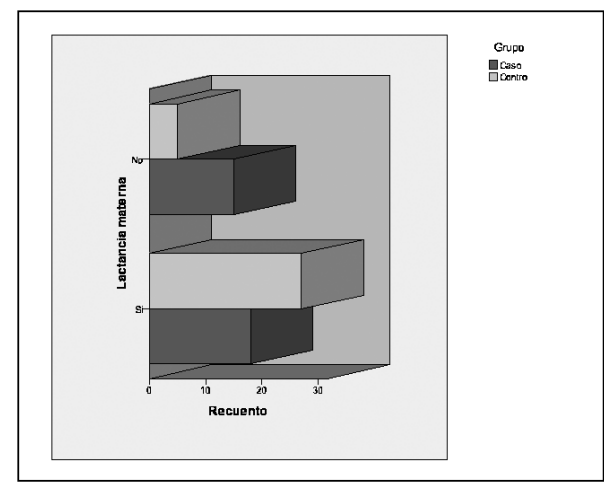

Figura 2. Distribución del número de niños con lactancia materna y sin ella en casos y controles $(p=0,045)$. OR: 0,222 (IC95\%: 0,069-0,719).

Por medio de un análisis de regresión logística se confirmó la asociación independiente de las variables LM, enfermedades de base e ingresos previos sobre el hecho de padecer bronquiolitis, con valores de OR: 0,25 (IC 95\%: 0,07-0,94), 4,53 (IC 95\%: $1,12-18,32)$ y 11,1 (IC 95\%: 1,22-100,52), respectivamente.

\section{DISCUSIÓN}

La enfermedad respiratoria aguda es la principal causa de enfermedad en la infancia, y supone un impacto de salud tanto en países industrializados como en vías de desarrollo, siendo una causa importante de mortalidad ${ }^{8}$. La bronquiolitis es la principal infección de vía respiratoria baja en lactantes.

En nuestro estudio hemos encontrado una distribución clásica: el $50 \%$ de pacientes tenía una edad inferior a 7 meses con leve predominio masculino, tal y como se describe en la literatura ${ }^{9,25}$. Supone una limitación en nuestro estudio el hecho de no haber tomado en consideración variables importantes como el número de hermanos que convivían en el hogar, antecedentes de patología respiratoria en los padres, así como la dermatitis atópica o la rinitis alérgica en familiares de primer grado.

Requirieron ingreso 7 de los pacientes, lo que supone un porcentaje muy superior al descrito en la literatura, con un 2-5\% de los niños de menos de 12 meses que requiere hospitalización ${ }^{4}$. Con respecto a los posibles factores de riesgo analizados, no hemos encontrado diferencias estadísticamente significativas con respecto a la edad gestacional. Sin embargo, la importancia de la prematuridad como factor de riesgo para el desarrollo de bronquiolitis aparece en numerosas publicaciones, tanto por presentar una incidencia aumentada, como por una mayor gravedad de la misma ${ }^{10,12-14}$.

En nuestro estudio no se han demostrado tampoco diferencias estadísticamente significativas en relación con la exposición al humo de tabaco en domicilio. Sin embargo, Mahabee-Gittens ${ }^{11}$ señala una prevalencia de fumadores en los hogares de niños afectos de asma y/o bronquiolitis de hasta el $41 \%$ de los casos. No existe ninguna duda de que la exposición al humo del tabaco medioambiental y el tabaquismo pasivo contribuyen a las infeccio- 
nes de las vías respiratorias inferiores en lactantes y niños pequeños en países en desarrollo y en las naciones industrializadas y constituye el principal contaminante ambiental al que están expuestos los niños ${ }^{10,11,15}$.

No hemos encontrado ningún estudio en la literatura médica que establezca una clara relación entre la exposición al humo de tabaco medioambiental y el riesgo en el lactante prematuro de hospitalización por infección por VRS. La naturaleza dudosa de las pruebas del papel del tabaquismo pasivo sobre la gravedad de la bronquiolitis por VRS ha propiciado en parte que la American Academy of Pediatrics (AAP) no tenga en cuenta firmemente el tabaquismo como un factor de riesgo cuando considera la profilaxis con palivizumab de lactantes nacidos prematuramente de 28 a 32 semanas de edad gestacional y menos de 6 meses antes de noviembre, prematuros de menos de 28 semanas de edad gestacional y 12 meses de edad, niños menores de 2 años diagnosticados de displasia broncopulmonar moderada-grave y en niños menores de 6 meses y cardiopatía congénita con compromiso hemodinámico (criterios estrictos siguiendo la recomendación de la guía SIGN) ${ }^{6}$.

En un estudio realizado por McConnochie et $\mathrm{al}^{16}$, a través de un análisis multivariado que incluyó el nivel socioeconómico, LM, el hacinamiento, el número de hermanos en el hogar y la existencia de antecedentes familiares de asma, se demostró un efecto significativo del tabaquismo pasivo en individuos con o sin antecedentes familiares de asma. Otros estudios prospectivos no han confirmado esta relación, aunque se diseñaron para examinar los factores de riesgo del desarrollo ulterior de asma, y no analizaron los diversos factores de riesgo de hospitalización para infección por $\mathrm{VRS}^{17}$.

El hábito tabáquico materno durante el embarazo parece asociarse con una mayor incidencia de bronquiolitis después del nacimiento ${ }^{10,26}$. Respecto a la relación establecida entre tabaquismo y LM, el estudio realizado por Chatzimichael et $\mathrm{al}^{8}$ determina que el tabaquismo materno incrementa el riesgo de infecciones del tracto respiratorio en niños alimentados al pecho durante menos de 6 meses, pero no si la LM se prolonga más allá del sexto mes de vida. Los autores destacan así que el papel protector de la LM es mayor en niños fumadores pasivos. Por otro lado, la asociación de historia familiar de asma y tabaquismo ambiental podría tener un efecto sumatorio en el riesgo de padecer bronquiolitis.

Numerosos estudios sugieren que la LM protege, confiriendo a través de inmunidad pasiva protección frente al VRS y otras enfermedades víricas, especialmente durante el primer mes de vida. El tiempo de lactancia inferior a 4 meses se asocia a un aumento de la probabilidad relativa de padecer un episodio severo y un mayor tiempo de hospitali- zación, encontrando el efecto indirecto que supone una disminución de la exposición al tabaco debido a que las madres fuman menos posiblemente por un mayor contacto del niño con la madre frente al padre ${ }^{13}$. Cuando la madre o ambos progenitores son fumadores, se incrementa el riesgo de sibilancias en los primeros 2 años de vida, pero esto no ocurre cuando el fumador es el padre. Destacan como conclusiones principales el aumento de prevalencia de bronquiolitis en lactantes expuestos al tabaco así como un aumento de su gravedad. La duración de la lactancia y la exposición al tabaquismo pasivo y activo podrían estar relacionadas con la severidad de la infección, así como con el tiempo de hospitalización. Se observó que niños con lactancia materna están protegidos frente al efecto del tabaquismo pasivo ${ }^{8}$.

La leche humana desempeña un destacado papel como nutriente óptimo para el recién nacido y el lactante pequeño (hasta los 12 meses de vida). La LM parece conferir protección inmunológica frente a las infecciones y los procesos alérgicos, y puede reducir la incidencia de enfermedades infecciosas en la infancia. El amamantamiento promueve los factores bioactivos, hormonas, factores de crecimiento, nutrientes específicos y factores estimulantes de la flora bacteriana, y produce la maduración de la mucosa gastrointestinal. Además, los niños con LM se exponen menos a los antígenos de la dieta complementaria o externa, confiriendo protección incluso después de finalizarla ${ }^{19}$.

El impacto de la LM sobre la salud de los niños ha sido ampliamente estudiado a través de la literatura médica. Recientes publicaciones aportan evidencias de los beneficios de la LM en la protección inmunológica y se asocia con tasas más bajas de hospitalización. En países en vías de desarrollo hay pocas dudas sobre su eficacia para disminuir la incidencia de estos procesos. Sin embargo, cuando estas investigaciones se realizan en países desarrollados, los resultados son contradictorios ${ }^{7,8}$. Posiblemente las diferencias a favor de la lactancia materna pueden no ponerse de manifiesto en países desarrollados si lo que se mide es la disminución de la mortalidad, y sí en otros en los que debido a las deficientes condiciones sanitarias y ambientales se ponen de manifiesto diferencias entre LM y artificial en términos de mortalidad por gastroenteritis aguda. Otro factor asociado podría deberse a la existencia de un número demasiado elevado de variables de confusión que es necesario controlar y que pueden oscurecer el posible efecto protector de la $\mathrm{LM}^{19}$.

En el año 1989 la Organización Mundial de la Salud (OMS) y la UNICEF hicieron una declaración conjunta para fomentar la LM. Estos organismos internacionales declaran que la LM forma parte natural e ideal de la alimentación del lactante y constituye una base biológica única para el desarrollo del niño. 
La Asociación Española de Pediatría aconseja la alimentación del niño con LM durante el primer semestre de vida y recomienda la puesta en marcha de programas para fomentarla ${ }^{20,21}$. Los programas de promoción en otros países han conseguido aumentos significativos de la LM, pero continúa habiendo dificultades en su prolongación durante un período suficiente para que proporcione los efectos beneficiosos deseados ${ }^{19}$. En nuestro país estudios recientes revelan unas tasas bajas de lactancia materna: aunque cerca del $90 \%$ de las madres la empiezan, al mes sólo continúa un 30\%; a los 3 meses más de la mitad de los niños toma lactancia artificial y a los 6 meses sólo un 10\% mantiene la lactancia materna ${ }^{22}$.

Es razonable especular que la leche materna puede brindar varios efectos sobre el desarrollo de las vías respiratorias y su posterior capacidad para combatir las infecciones. Los factores específicos de nutrición inmunorreguladora e inmunomoduladora en la leche materna podrían promover la maduración de la capacidad inmune del bebé ${ }^{23,24}$. Bryan et al ${ }^{24}$ trataron de analizar los factores inmunomoduladores y la activación del sistema inmune a través del análisis de la leche materna de niños hospitalizados diagnosticados de bronquiolitis grave. Observaron cambios que afectan a la célula no sólo en los sitios de activación, tales como la lesión del epitelio, sino también en sangre periférica. El análisis determinó un aumento en las células monocucleares, en los niveles séricos de IL-4 e INF-C. Adultos con atopia presentan en comparación niveles más elevados de Linfocitos T colaboradores Th2, citocinas, IL-4, IL-5 y reducidos de citocinas Th1, IL-12. Dada la alta contagiosidad del Virus Respiratorio Sincitial, el estrecho contacto establecido entre madre-lactante provocaría una respuesta inmunológica, una infección subclínica para el adulto, dando lugar a la afluencia de leucocitos activados en la glándula mamaria, transmitiéndose al bebé a través de la leche. Tras una segunda exposición al VRS, con alteración en la respuesta de citocinas y disminución en los niveles de TH1, TH2 y IL-10, producida por macrófagos alveolares, se elevarían en respuesta a la infección, por lo que sería esperable encontrar niveles elevados en la leche materna, encontrando un descenso considerable en los niveles que podría ser el responsable en el desarrollo de sibilancias recurrentes y asma posterior.

En nuestro estudio la LM ha demostrado ser factor protector frente a la bronquiolitis en los lactantes de 0 a 24 meses de vida atendidos en un centro de salud, con un $45 \%$ de los casos sin LM frente a un $15,6 \%$ de los controles. Estos resultados subrayan la importancia de promover la lactancia exclusiva hasta los seis meses de vida, no sólo por la prevención de enfermedades infecciosas, sino también por el curso de menor agresividad de la patología respiratoria ${ }^{23,26}$.
La LM es un claro factor protector, considerándose como la mejor elección para el recién nacido en relación a su crecimiento y desarrollo, por lo que su promoción será sin duda una de las actividades más eficaces para evitar la bronquiolitis en nuestro medio. La presencia de patología de base u hospitalización previa predispone al desarrollo de bronquiolitis y es, por tanto, fundamental el seguimiento estrecho de estos lactantes de riesgo en época de epidemia.

\section{BIBLIOGRAFÍA}

1. Uberos Fernández J. Bronquiolitis aguda: Actualización sobre nuevas evidencias en su manejo. Sociedad Española de Pediatría Extrahospitalaria y Atención Primaria. Disponible en: http://www.sepeap.org/archivos/ revisiones/neumologia/ bronquiolitis.htm (con acceso el 4-2-2010).

2. Callén Blecua M, Torregrosa Bertet MJ, Bamonde Rodríguez L y Grupo de Vías Respiratorias. Guía Rápida del Protocolo de Bronquiolitis. Diagnóstico y tratamiento en Atención Primaria. Protocolo del GVR (publicación P-GVR-4-gr). Disponible en: http://www.aepap.org/gvr/protocolos.htm (con acceso el 4-2-2010).

3. McNamara PS, Flanagan BF, Smyth RL, Hart A. Impact of human Metapheumovirus and respiratory syncytial virus co-infection in severe bronchiolitis. Pediatr Pulmonol. 2007; 42:740-3.

4. Vicente D, Montes M, Cilla G, Pérez Yarza EG, Pérez-Trallero E. Hospitalization for respiratory syncytial virus in the pediatric population in Spain. Epidemiol Infect. 2003; 131: 867-72.

5. Sardón O, Korta J, Pérez-Yarza E. Bronquiolitis. An Pediatr Contin. 2007; 5:332-42.

6. Diagnosis and Management of Bronchiolitis.Clinical Practice Guideline. Pediatrics. 2006; 118:1774-93.

7. Van Esso DL. Lactancia materna e infecciones respiratorias. Aten Primaria. 2002; 29:276-7.

8. Chatzimichael A, Tsalkidis A, Cassimos D, Gardikis S. The role of breadsfeeding and passive smoking on the development of severe bronchiolitis in infants. Minerva Pediatrics. 2007; 59:199-206.

9. García Martín FJ, Moreno Pérez D. Protocolos diagnósticos y terapéuticos en pediatría. Disponible en: http//www. aeped.esprotocolos/infectología/04.Bronquiolitis.pdf (con acceso el 4-2-2010).

10. Cano Fernández J, Zabaleta Camino C, De la Torre Montes de Neira E, Yep Chullen GA, Melendi Crespo JM, Sánchez Bayle M. Tabaquismo pasivo prenatal y posnatal y bronquiolitis. An Pediatr (Barc). 2003; 58:115-20.

11. Mahabee-Gittens M. Smoking in parents of children with asthma and bronchiolitis in a pediatric emergency department. Pediatr Emerg Care. 2002; 18:4-7.

12. Alba Moreno F, Alsina Donadeu J. Estudio clínico-epidemiológico de las enfermedades del tracto respiratorio inferior con sibilancias en menores de 2 años y factores de riesgo asociados. An Esp Pediatr. 1999; 50:379-83.

13. De la Torre MJ, Martín-Calama J, Hernández-Aguilar MT, Spanish Committee on Human Lactation, Spanish Pediatric Association. Breast-feeding in Spain. Public Health Nutr. 2001; 4:1347-51.

14. Gómez Sanchiz M, Baeza Berruti JE, González Mateos 
JA, Avila Martín-Gil O. Prevalencia y características de la lactancia maternal en el medio rural. Aten Primaria. 1997; 19:318-22.

15. Gurkan F, Kiral A, Dagli E, Karakoç F. The effect of passive smoking on the development of respiratory syncytial virus bronchiolitis. Eur J Epidemiol. 2000; 16:465-8.

16. McConnochie KM, Roghmann KJ. Parental smoking, presence of older siblings, and family history of asthma increase risk of bronchiolitis. Am J Dis Child. 1986; 140:806-12.

17. Simoes EA. Tabaquismo materno, asma y bronquiolitis: ¿asociación clara o pruebas dudosas? Pediatrics (Ed Esp). 2007; 63:342-4.

18. González Caballero D, González Pérez Yarza E. Bronquiolitis aguda: bases para un protocolo racional. An Esp Pediatr. 2001; 55:355-64.

19. Carratalá Munera MC, Gascón Pérez E, Raga Ortega M. ¿Es la lactancia materna un factor de protección ante los procesos infecciosos? Estudio de casos y controles. Aten Primaria. 2005; 35:140-5.

20. Bryan DL, Hart PH, Forsyth KD, Gibson RA. Inmunomodulatory constituents of human milk change in response to infant bronchiolitis. Pediatr Allergy Inmunol. 2007; 18:495-502.
21. OMS-UNICEF. Sobre la alimentación del lactante y del niño pequeño. Reunión conjunta OMS-UNICEF. Ginebra: OMS; 1989.

22. Comité de Lactancia Materna de la Asociación Española de Pediatría. Informe técnico sobre la lactancia materna en España. An Esp Pediatr. 1999; 50:333-40.

23. Lasarte Velillas JJ, Hernández Aguilar M.T: Preguntas más frecuentes. Rev Pediatr Aten Primaria. 2009; 11(Supl 17):s405-s414.

24. Dornelles C, Piva JF, Marostica P. Nutricional status, breastfeeding, and evolution of infants with Acute Viral Bronchiolitis. J Health Popul Nutr. 2007; 25:336-43.

25. Seiden JA, Scarfone RJ. Bronchiolitis: an evidence-based approach to management. Clin Pediatr Emerg Med. 2009; 10:75-81.

26. Pérez Tarazona S, Alfonso Diego J, Amat Madramany A, Chofre Escrihuela L, Lucas Sáez E, Bou Monterde R. Incidencia y factores de riesgo de bronquitis sibilantes en los primeros 6 meses de vida en una cohorte de Alzira (Valencia). An Pediatr (Barc). 2010; 72:19-29. 